\title{
Preparation of Au-Ag composite Nanoparticles by Pulsed Laser Ablation in Water for controlling of AIP enzyme Activity in human blood
}

\author{
Rasha B. Rashid
}

\begin{abstract}
In this work, a colloidal suspension aqueous solution of Au/Ag composite nanoparticles by pulsed laser ablation method was obtained. Q-switched $N d: Y A G, \lambda=1064 \mathrm{~nm}, 532 \mathrm{~nm} 10 \mathrm{~ns}$ pulse duration and E=100$900 \mathrm{~mJ}$ and by using pure Ag and Au metal plates immersed in pure water. The optical extinction spectra have been obtained in the Plasmon resonance region. The results are supported by X-ray data and transmission electron microscopy. UV-VIS absorption spectra of produced solution show a sharp peak around $400 \mathrm{~nm}$, $525 \mathrm{~nm}$ indicating the produced Ag and Au nanoparticles respectively with a narrow size ranging from (5 to 45) $n m$ for Ag NPs, (8-55)nm for Au NPs and (10-170)nm for Au/Ag composite NPs with almost spherical shape Xray data indicate the fcc (face-centered cubic) structure.This work presents the effect of number of pulses on the properties of the prepared Au, Ag and Au-Ag alloy NPs, also included a study of the biomedical effects of Au, $A g, A u-A g$ alloy NPs on the activity of Alkaline Phosphatase enzyme in human blood.
\end{abstract}

Keywords: Nanoparticles, Au, Ag, composite, coer-shell, aqueous, Nd: YAG

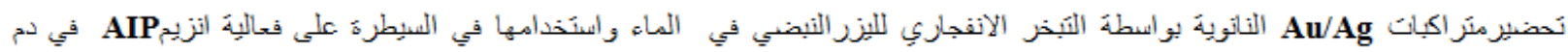

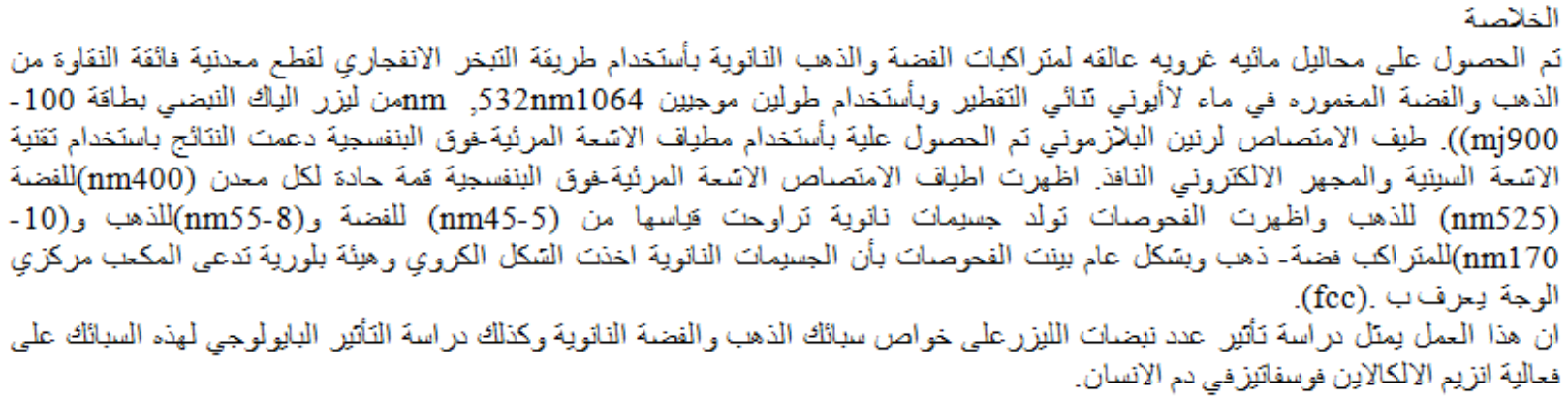

\section{Introduction}

Nanoscience has a great and continuous growing of attention due to growing of its applications in all fields of sciences. As well as, the preparation methods of nanoparticles was expanded to consist chemical, physical, biological and engineering methods. One of them methods in laser ablation method, which produce colloidal nanoparticles in different kinds of solutions [1-3]. The properties of Nanoparticles are mainly controlled with controlling their shape, size, composition, crysallinity and structure, while the properties of the matrix in which NPs are dispersed in, controller the properties of the relative nanocomposites [4-5]. The wellknown unique optical, electronic and catalytic properties of nanocrystal alloys cause more interesting in this field so this importance differs from those of the monometallic constituents. Methods of preparation for $\mathrm{Au} / \mathrm{Ag}$ Alloy particles have been known since several years, One of these methods was evaporation of $\mathrm{Au} / \mathrm{Ag}$ bulk solid solutions[6] also the simultaneous reduction of two different metal salts in water form M1/M2 alloy nanoparticles with few nanometers in diameter [4]. Laser irradiation processes got more attention in the last few years in the preparation of metal colloids. Mafune et.al.[7-8]. Showed that laser ablation of transition metal targets can be performed in liquid media, and proved the possibility of producing metallic colloids without the presence of pollutant ions at the end of the formation process. Also it is possible to extend this procedure to use different liquids other than water as proved by Compagnini and Co-Work [9-10]. While Amendola et.al.[11] controlled the aggregation of metallic clusters by re- irradiation. Lee et al.[12] synthesized Au/Ag NPs as colloidal solution by laser ablation of bulk solid solutions in water. In this process laser radiation applied directly to a gold-silver colloidal mixture to form alloy nanoparticles [13]. Deng et al.[14] studied the optical properties of the colloidal alloy so a number of different considerations has been done based on the results also they studied the Plasmon resonance behavior which strongly depends on the composition of the mixed clusters which confirms the presence of core-shell structure or the formation of true solid solution. 
Alkaline phosphates (ALP) is present in a number of tissues including liver, bone, intestine, and placenta. Serum ALP is of interest in the diagnosis of 2 main groups of conditions-hepatobiliary and bone diseases which are associated with increased osteoblastic activity. The main site of new enzyme synthesis is the hepatocytes adjacent to the biliary canaliculi.

\section{Experimental}

Laser ablation method has been used to prepare gold and silver colloids separately fig. (1-A) shows the experimental setup of Pulse Laser Ablation in Liquid (PLAL) system. In each case silver and gold plates (>99.99\% purity) were placed at the bottom of plastic vessel contains $1 \mathrm{ml}$ of double distilled and deionised water (DDDW). Ablation done by irradiation the metal plate with 80 pulses of $700 \mathrm{~mJ} \mathrm{Nd:YAG} \mathrm{laser} \mathrm{(1064nm)}$ operating at $1 \mathrm{~Hz}$ and pulse width of $10 \mathrm{~ns}$. The laser beam has been focused by a lens having a focal length of $100 \mathrm{~mm}$, the spot diameter of the laser beam on the metal surface has been fixed at $1.5 \mathrm{~mm}$ to obtain a power density around $40 \mathrm{~J} / \mathrm{cm} 2$ on the sample. Au-Ag composite was prepared by irradiation Au target in fresh prepared Ag NPs colloidal solution, with the same preparation method of pure Au NPs and Ag NPs after 80pulses of 1064nm Nd:YAG laser. Figure (1-B) shows the experimental setup for composite preparation. The Au target was released and the colloidal mixture solution was irradiated with a period of 100 pulses of $532 \mathrm{~nm}$ Nd:YAG laser. The formed colloid was mixed and then irradiated with $(532 \mathrm{~nm}) \mathrm{Nd}$ : YAG laser with the same properties of the $1064 \mathrm{~nm}$ which used a above. All colloidal solutions and the irradiated colloidal mixture have been immediately characterized by UV-VIS. Spectrophotometric technique using SP-3000 Pluse CE OPTMA spectrometer TOKYO, JAPAN, in range 300-600 nm. Some drops of the colloidal solutions and the irradiated mixture have been deposited onto a glass substrates to obtain the X-ray spectra using (XRD, SHIMADZU, 6000). The principle of the enzyme is kinetic and depends on substrate metal. Generally it is working according to the following reaction.

\section{p-Nitrophenylphosphate $+\mathrm{H} 2 \mathrm{O} \rightleftarrows$ Phosphate+p-nitrophennol}

\section{Results and general discussion}

Fig. 2 shows the nanoparticales solutions of $\mathrm{Ag}$ NPs, $\mathrm{Au}$ NPs, $\mathrm{Au} / \mathrm{Ag}$ mixture NPs and $\mathrm{Au} / \mathrm{Ag}$ composite NPs. They are colored, the faint yellow and faint pink colloidal solution of silver and gold NPs respectively, are obtained after laser irradiation of $1064 \mathrm{~nm}, 80$ pulses and also to obtained mixture NPs. The optical colors are attributed to the formation of metal nanoparticles as they scatter bright intense colors. Preparing composite NPs from the prepared solution was obtained by using Nd:YAG laser (532nm), 100pulses. This indicates the presence of NPs by the changing of solution color. Fig. (3-A) shows the Plasmon resonance spectra of pure metal colloidal solution of gold NPs and silver NPs, mixture of Au/Ag NPs as prepared and mixture of Au/Ag NPs irradiated with100 pulses of 532nm Nd:Yag laser. After irradiation with 100 pulses of $532 \mathrm{~nm} 700 \mathrm{mj}$ absorption peak of Ag NPs was fixed at $405 \mathrm{~nm}$ but the absorption peak of Au NPs shift to $515 \mathrm{~nm}$ (blue shift) and this due to formation of core-shell structures. While, Fig. (3-B) shows the effect of a period of shots (532nm, 700mj, 100 pulses each step) on Plasmon resonance spectra of Au/Ag NPs mixture.

As shown in Fig. (3-B) the evolution of the plasmon resonance spectra of the mixture of Au NPs and Ag NPs in the prepared colloidal solution in boiled DDDW with laser irradiation time (No. of pulses). Before irradiation, the plasmon resonance peaks of Ag and Au are observed at 405 and 520nm, respectively. The rather high value of $405 \mathrm{~nm}$ indicates that the diameter of Ag particles is rather high, After irradiation with a period of 100 pulses each step the plasmon resonance peak of Ag NPs was fixed but the peak corresponding to Au shifted slightly to $510 \mathrm{~nm}$ (blue shift) this shift confirms that $\mathrm{Ag}$ and $\mathrm{Au}$ particles have come into contact.A consideration immediately comes out intensities and band shape modifications of bimetallic colloids are at various but comparable number of $532 \mathrm{~nm}$ pulses. The spectral absorption position of the Ag Plasmon resonance maximum remains fixed, While that of the Au monotonically blue shifts at higher energies with increasing irradiation pulses. This is a clear indication that $\mathrm{Au}$ core $\mathrm{Ag}$ shell nanoparticles are constituted by variable composition objects, with a continuous inclusion in the core of silver atoms coming from the shell. Several authors $[15,16,17]$ reported the fact that the colloidal dispersion of metallic particles composed by an Au-Ag solid solution is obtained at long irradiation times, is proven by the existence of this single plasmon resonance, ruling out the possible formation of core-shell structure.

Fig. 4 presents TEM images of prepared Ag NPs, Au NPs and Au-Ag composite NPs. The size distribution of nanoparticles shows that the size of silver nanoparticles ranged from 5-45 $\mathrm{nm}$ and the frequency percent decreases with the increasing of the nanoparticle size. For gold nanoparticles it ranged from 8-55 nm, and the distribution for gold shows that the frequency percent increases with the increasing nanoparticle size. For $\mathrm{Au} / \mathrm{Ag}$ composite the size ranged from 10-170nm. Comparison with TEM images of pure metal nanoparticles, which are all dark colored and Au/Ag core-shell nanoparticles yield similar looking TEM images with dark centers in lighter surrounding material. The TEM image of composite shows core-shell structure of 
Au-Ag NPs.Morphology and crystal structure of Au NPs, Ag NPs and Au-Ag composite NPs was examined using X-Ray diffraction spectrophotometer (XRD, SHIMADZU, 6000). Fig. 5 shows XRD patterns each pattern consist of one peak, it is at $(2 \Theta=38.43)$ for $\mathrm{Ag},(2 \Theta=38.46)$ for $\mathrm{Au}$ and $(2 \Theta=38.56)$ for $\mathrm{Au}-\mathrm{Ag}$ composite. The appearance of only one peak in each pattern means that all prepared NPs are in single crystal shape with (111) miller indices, which means all prepared nanoparticles are with fcc (face-centered cubic) structure.The concentration of ablated Ag and Au nanoparticles in suspension solution was obtained using Atomic Absorption Spectrophotometer (AAS).A linear relationship of metal NPs concentration with number of pulses and the relationship between concentration and Plasmon resonance absorbance shown in fig.(6-A) for Ag NPs and fig. (6-B) for Au NPs. This figure shows that the concentration increased with increasing number of pulses also the absorbance increased with increasing concentration for both cases. The table (1) show the activity of AIP had changed in presence different NPs. In presence Au NPs the activity increase from 21 to 52 that means the activity was146\%. While in presence Ag NPs the activity decreases from 21 to 17 that's means Ag NPs inhibited the AIP enzyme to $19.5 \%$. The same accrued when we added composite (Ag- $\mathrm{Au}$ ) here we see the inhibition become $78.53 \%$.

\section{Conclusions}

We conclude that the preparation of $\mathrm{Au} / \mathrm{Ag}$ composite nanoparticles with well-defined size and shape can be obtained by laser irradiation of mixture of $\mathrm{Ag}$ and $\mathrm{Au}$ colloides with no additives, such as solvents, surfactants or reducing agents are needed in the procedure. Optical measurements of colloidal nanoparticles exhibit single maximum optical extinction at $400 \mathrm{~nm}$ and $525 \mathrm{~nm}$, which are related to surface plasmon resonance of silver and gold nanoparticles respectively. It is well known that the formation efficiency of PLAL was quantified in term of the absorbance peaks as well as of the concentration of ablated metal nanoparticles obtained by atomic absorption spectroscopy. Study of the ability of NPs to inhibit or activate alkaline phosphates was shown activation with Au NPs and inhibition with Ag NPs and Au/Ag composite NPs.

\section{References}

[1]. W. Eberhardt (Clusters as new materials) Surf. Sci. 500 (2002) 242.

[2]. A.W. Castleman, K.H. Bowen (Clusters: Structure, Energetics, and Dynamics of Intermediate States of Matter) J. Phys. Chem. 100 (1996) 12911

[3]. G. Compagnini, L. D’Urso, O. Puglisi, Mater (Mass and velocity distributions of supersonic cluster beams) Sci. Eng. C 26 (2006) 1082 .

[4]. S. Link, M.A. El-Sayed ( Spectral properties and relaxation dynamics of surface plasmon electronic oscillations in gold and silver nanodots and nanorods) J. Phys. Chem. B 103 (1999) 8410, 1999.

[5]. J. Roiz, A. Oliver, E. Munoz, L. Rodriguez-Fernandez, J.M. Hernandez, J.C. Cheang-Wong (modification of the optical properties of Ag-implanted silica by annealing in two different atmospheres) J. Appl. Phys. 95 (2004) 1783.

[6]. G.C. Papavassiliou ( Surface plasmons in small Au-Ag alloy particles) J. Phys. F: Met. Phys. 6 (1976) L103.

[7]. F. Mafune, J. Kohno, T. Takeda, T. Kondow ( Full physical preparation of size-selected gold nanoparticles in solution: Laser ablation and laser-induced size control) J. Phys. Chem. B 106 (2002) 7575.

[8]. F. Mafune, J. Kohno, Y. Takeda, T. Kondow, H. Sawabe, (Formation and Size Control of Silver Nanoparticles by Laser Ablation in Aqueous Solution), J. Phys. Chem. B 104 (2000) 9111.

[9]. G. Compagnini, A.A. Scalisi, O. Puglisi (Ablation of noble metals in liquid: a method to obtain nanoparticles into a thin polymeric film) Phys. Chem. Chem. Phys. 4 (2002) 2787.

[10]. A.Scalisi,G. Compagnini, O. Puglisi (Production of gold nanoparticles by laser ablation in liquid alkanes) J.AppI.Phys. 94 (2003) 7874 .

[11]. V. Amendola, S. Polizzi, M. Meneghetti (Laser Ablation Synthesis of Gold Nanoparticles in Organic Solvents) J. Phys. Chem. B 110 (2006) 7232

[12]. I. Lee, S.W. Han, K. Kim (Production of Au-Ag alloy nanoparticles by laser ablation of bulk alloys) Chem. Commun. 18 (2001) 1782 .

[13]. Y. Chen, C. Yeh (A new approach for the formation of alloy nanoparticles: laser synthesis of gold-silver alloy from gold-silver colloidal mixtures) Chem. Commun. 4 (2001) 372.

[14]. Z. Peng, B. Spliethoff, B. Tesche, T. Walther, K. Kleinermanns (Laser-assisted synthesis of Au-Ag alloy nanoparticles in solution) J. Phys. Chem. B 110 (2006) 2549.

[15]. S. Link, M. A. El-Sayed (Size and temperature dependence of the plasmon absorption of colloidal gold nanoparticles) J. Phys. Chem. B 103, 4212 (1999)

[16]. M. Moskovits, I. Srnova-Sloufova, B. Vlckova (Bimetallic Ag-Au nanoparticles: Extracting Meaningful Optical Constants from the Surface-plasmon Extinction Spectrum) J. Chem. Phys. 116 (2002) 10435

[17]. Huazhong Shi, Lide Zhang, and Weiping Cai (Composition modulation of optical absorption in AgxAu1-x alloy nanocrystals in situ formed within pores of mesoporous silica), J. Appl. Phys. 87 (2000) 1572. 
A

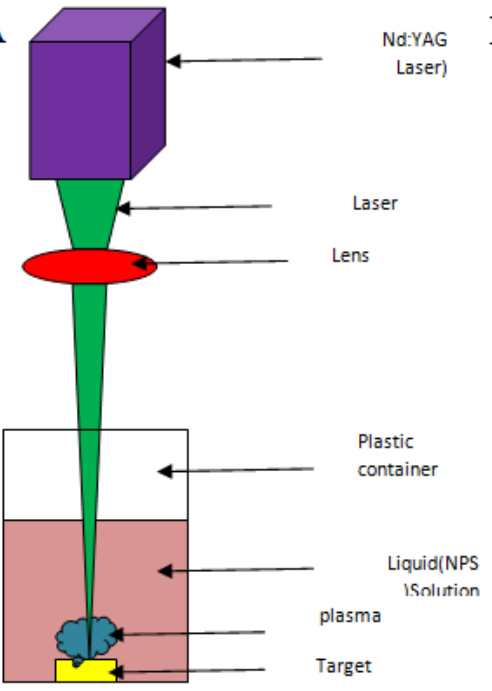

$\mathrm{B}$

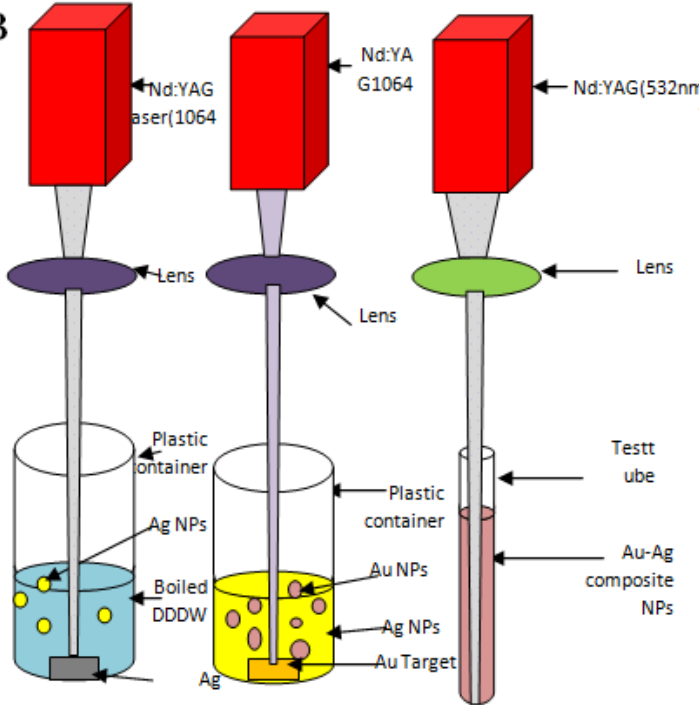

Fig. (1) A- Experimental setup for nanoparticles synthesis, by laser ablation technique, B- (Au-Ag) Composite Preparation steps.

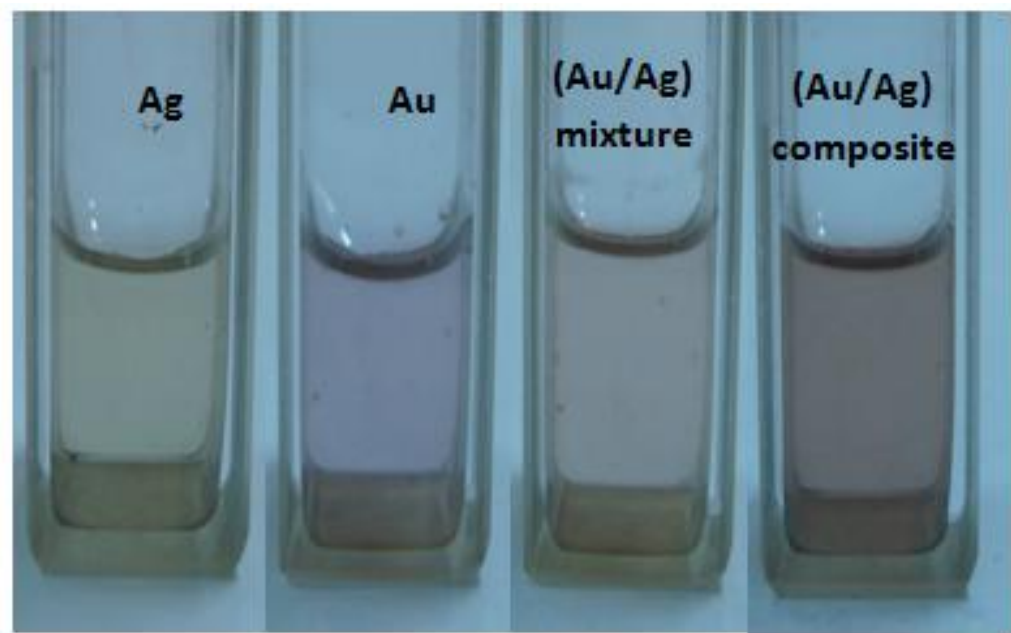

Fig.(2) Photograph of the final products of silver, gold, mixture and Au/Ag composite NPs solutions prepared by PLAL.

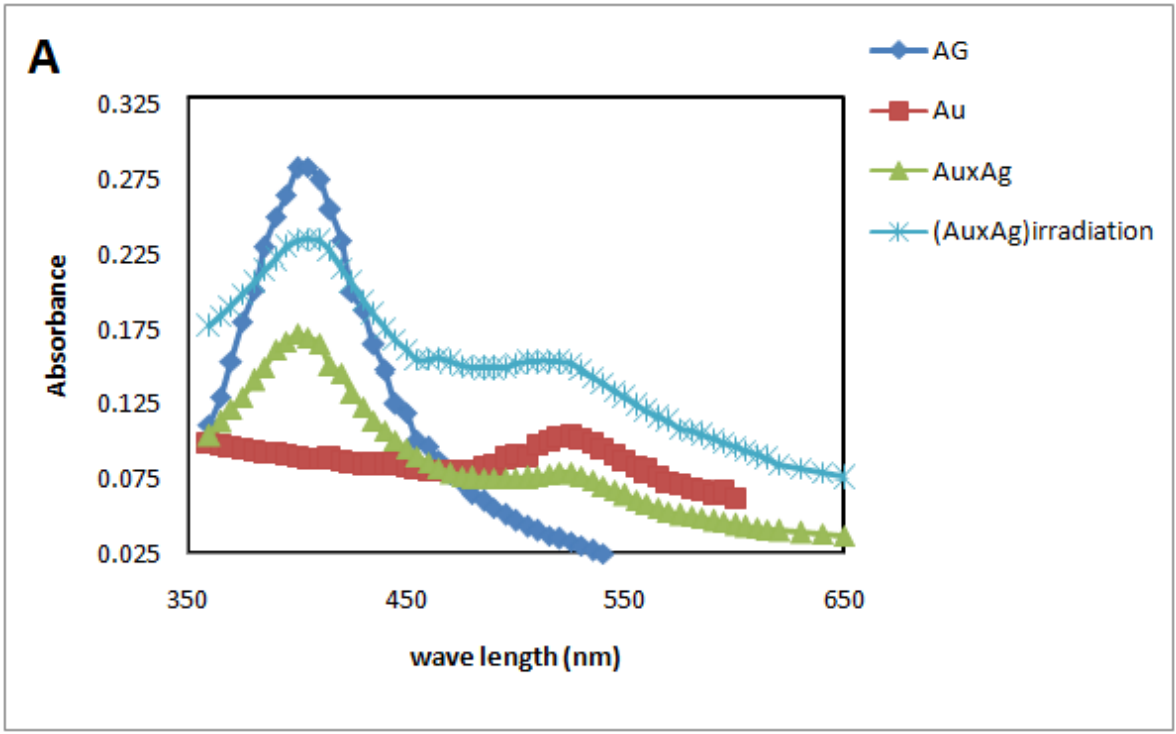




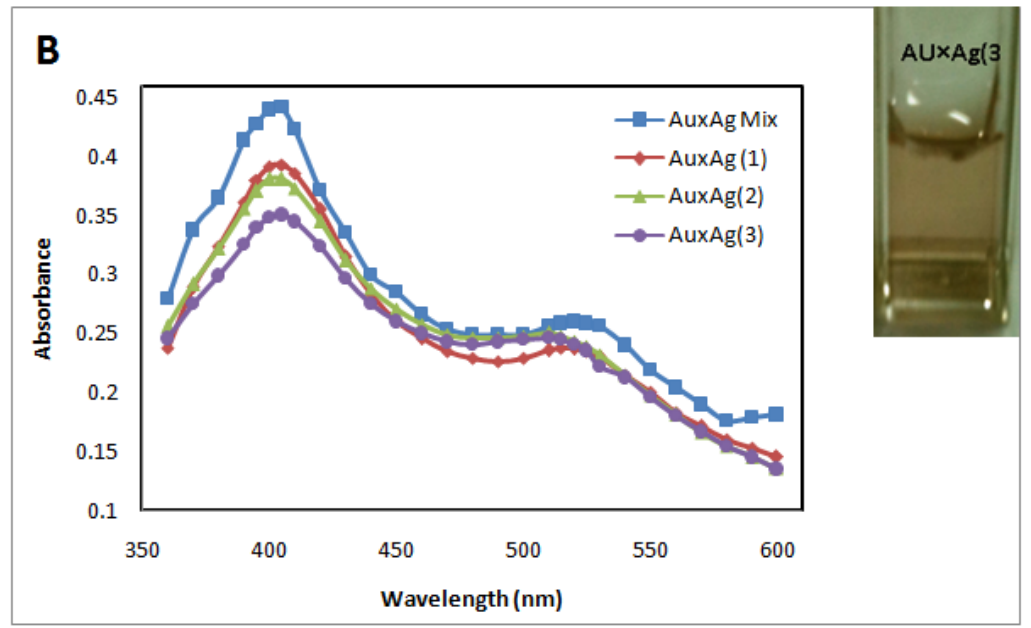

Fig. (3) Plasmon resonance spectra of (A) pure Ag NPs, Au NPs, mixture of Au/Ag NPs and irradiated Au/Ag NPs mixture ( $\lambda=532 \mathrm{~nm}$, laser shots of 100 pulses and $E=700 \mathrm{~mJ})$. (B) The effect of laser shoots (100 pulses, $700 \mathrm{Mj}$ each step)on $\mathrm{Au} / \mathrm{Ag}$ core-shell absorption peaks.
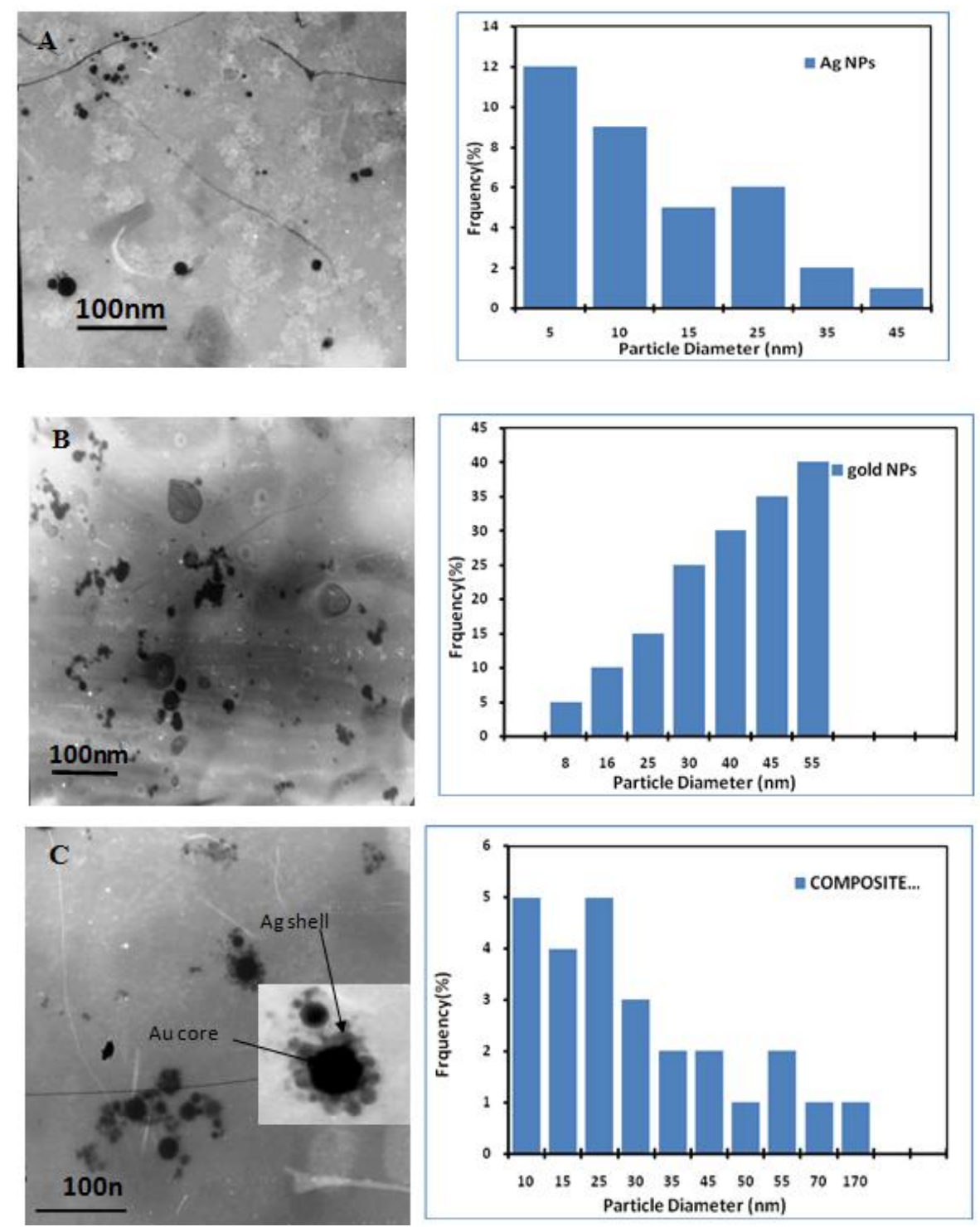

Fig. (4)TEM images and size distributions of the (A) silver, (B)gold, (C) composite nanoparticles, produced by laser ablation of target plate immersed in DDDW. 


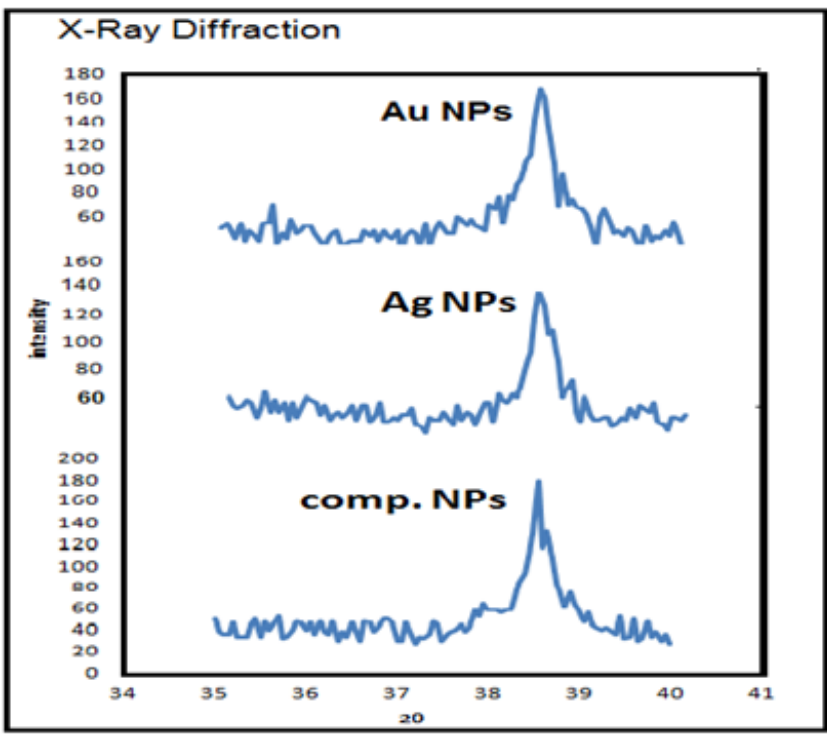

Fig. (5) XRD patterns of Ag, Au, Au-Ag composite NPs synthesized by laser ablation in $8 \mathrm{ml}$ DDDW.

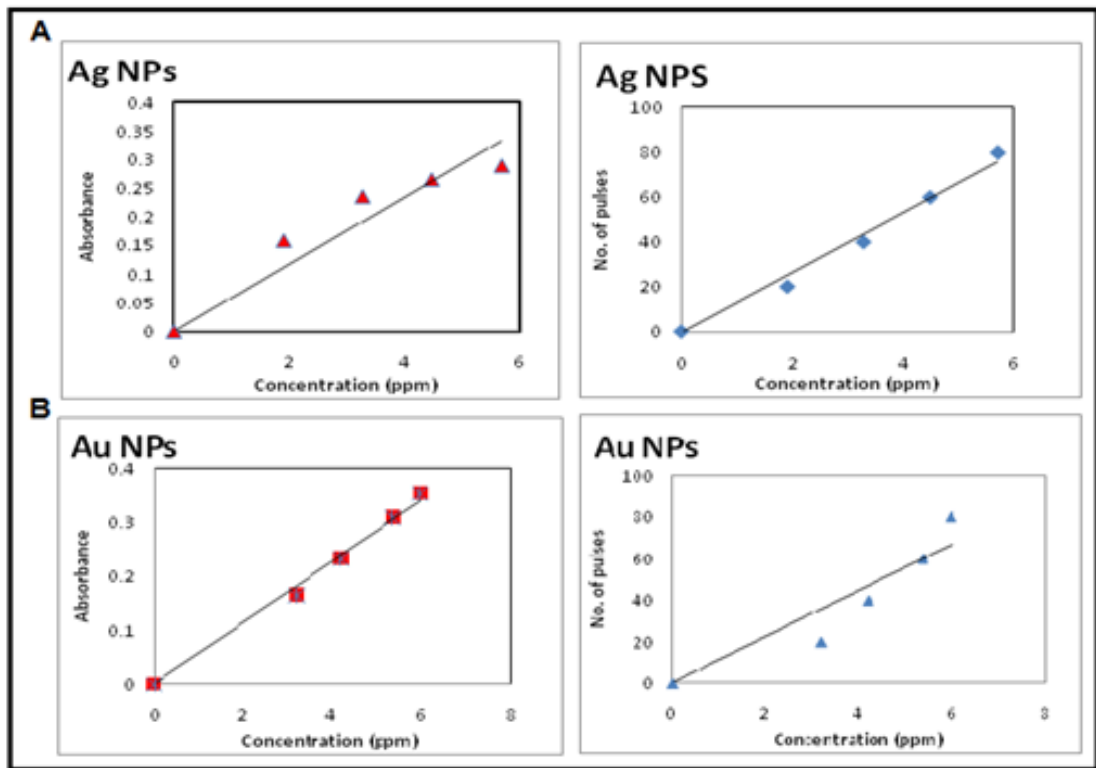

Fig.(6) Amount of ablated NPs a against number of pulses and absorbance. (A) for Ag NPs, (B) for Au NPs.

Table (1) show the activity of AIP

\begin{tabular}{|c|c|c|c|c|}
\hline \multirow{2}{*}{ Sample } & \multicolumn{2}{|c|}{ Activity U/L } & Inhibition\% & Activation\% \\
\cline { 2 - 3 } & $(-\mathbf{N P})$ & $(+\mathbf{N P})$ & & \\
\hline Gold & 21.33 & 52.64 & $\cdots \cdots \cdots$ & 146.79 \\
\hline Silver & 21.33 & 17.17 & 19.50 & $\ldots \ldots \ldots$ \\
\hline Composite & 21.33 & 4.58 & 78.53 & $\cdots \cdots$ \\
\hline
\end{tabular}

